\title{
BMJ Open Prevalence and incidence of kidney diseases leading to hospital admission in people living with HIV in France: an observational nationwide study
}

\author{
Magali Louis, ${ }^{1,2}$ Jonathan Cottenet, ${ }^{3}$ Arnaud Salmon-Rousseau, ${ }^{1}$ Mathieu Blot, \\ Pierre-Henri Bonnot, ${ }^{2}$ Jean-Michel Rebibou, ${ }^{2}$ Pascal Chavanet, ${ }^{1,4}$ \\ Christiane Mousson, ${ }^{2}$ Catherine Quantin, ${ }^{3}$ Lionel Piroth ${ }^{\circledR 1,4}$
}

To cite: Louis M, Cottenet J, Salmon-Rousseau A, et al. Prevalence and incidence of kidney diseases leading to hospital admission in people living with HIV in France: an observational nationwide study. BMJ Open 2019;9:e029211. doi:10.1136/ bmjopen-2019-029211

- Prepublication history for this paper is available online. To view these files, please visit the journal online (http://dx.doi. org/10.1136/bmjopen-2019029211).

Received 17 January 2019 Revised 27 March 2019 Accepted 3 April 2019

\section{Check for updates}

C Author(s) (or their employer(s)) 2019. Re-use permitted under CC BY-NC. No commercial re-use. See rights and permissions. Published by BMJ.

${ }^{1}$ Infectious Diseases

Department, University Hospital, Dijon, France

${ }^{2}$ Nephrology, University Hospital, Dijon, France

${ }^{3}$ CHRU Dijon, Service de Biostatistique et d'Informatique Médicale (DIM), Université de Bourgogne, Dijon, France ${ }^{4} \mathrm{CIC}$ 1432, INSERM, Dijon, France

Correspondence to Dr Lionel Piroth; lionel.piroth@chu-dijon.fr

\section{ABSTRACT}

Objectives To describe hospitalisations for kidney disease (KD) among people living with HIV (PLHIV) in France and to identify the factors associated with such hospitalisations since data on the epidemiology of KD leading to hospitalisation are globally scarce.

Design Observational nationwide study using the French Programme de Médicalisation des Systèmes d'Information database.

Setting France 2008-2013.

Participants Around 10862 PLHIV out of a mean of 5210856 patients hospitalised each year. All hospital admissions with a main diagnosis code indicating KD (International Classification of Diseases, 10th revision codes, N00 to -N39) were collected.

Main outcome measures The prevalence and incidence of KD leading to hospital admission in PLHIV and the associated risk factors.

Results The prevalence of patients hospitalised for KD was 1.5 higher in PLHIV than in the general population, and increased significantly from $3.0 \%$ in 2008 to $3.7 \%$ in $2013(p<0.01)$. The main cause of hospitalisation for KD was acute renal failure (ARF, 25.4\%). Glomerular diseases remained stable $(6.4 \%)$ throughout the study period, focal segmental glomerulosclerosis being the main diagnosis (37.6\%). Only $41.3 \%$ of patients hospitalised for glomerular disease were biopsied. The other common motives for admission were nephrolithiasis (22.1\%) and pyelonephritis (22.6\%). The 5-year cumulative incidence of KD requiring hospitalisation was $5.9 \%$ in HIV patients newly diagnosed for HIV in 2009. Factors associated with a higher risk of incident $\mathrm{KD}$ requiring hospitalisation were cardiovascular disease (HR 3.30, 95\% Cl 1.46 to 7.49 ), and, for female patients, AIDS (HR 2.45, 95\% Cl 1.07 to 5.58). Two-thirds of hospitalisations for incident ARF occurred in the first 2 years of follow-up.

Conclusions Hospital admission for KD is more frequent in PLHIV than in the general population and increases over time. ARF remains the leading cause. Glomerular diseases are infrequently documented by renal biopsies. Older patients and those with cardiovascular disease are particularly concerned.
Strengths and limitations of this study

- This study is the first to focus on hospital admissions for kidney disease (KD) in a large population of people living with HIV.

- The exhaustive nature of our data ensures that the results are representative of all hospitalisations for $\mathrm{KD}$ in France.

- The main limitation is the use of administrative codes, even though they are based on the clinical judgement of physicians and on laboratory values.

- It was also not possible to provide accurate characteristics of the HIV infection.

\section{INTRODUCTION}

In 2015, 36.9million people around the world and about 130000 in France were living with HIV. The introduction of highly active antiretroviral therapy (HAART) has dramatically decreased mortality and significantly improved the quality of life of people living with HIV (PLHIV). However, an increase in the incidence of comorbidities, including kidney disease $(\mathrm{KD})$, has been observed. ${ }^{12}$ Several studies have shown that acute renal failure (ARF) was three to four times more frequent in PLHIV than in the general population $^{34}$ and that its incidence did not decrease as HAART became more common. ${ }^{5}$ $\mathrm{ARF}$, in the general population and PLHIV, has also been associated with longer hospital stays and higher costs, and with a risk of progression to chronic KD (CKD).$^{6-8}$

The incidence of CKD is reportedly higher in PLHIV than in people not infected with HIV. ${ }^{9}$ Its prevalence has been estimated at between $1 \%$ in the Data collection on Adverse events of Anti-HIV Drugs (DAD) study ${ }^{10}$ and $4.9 \%$ in a French cohort of 2588 patients. ${ }^{11}$ The proportion of HIV-associated nephropathy (HIVAN) has significantly 
fallen, ${ }^{12}$ while that of other KDs, such as classical focal segmental glomerulosclerosis (FSGS) or HIV-associated immune complex KD (HIVICK), has increased. ${ }^{13}$ The increase in the incidence of CKD could also be related to the nephrotoxicity of some antiretroviral drugs or to the increased frequency of comorbidities, such as diabetes, hypertension, cardiovascular disease, or hepatitis C or B coinfection. ${ }^{6} 1014$

However, to our knowledge, no studies have dealt with the epidemiology of $\mathrm{KD}$ leading to hospitalisation in PLHIV in the recent HAART era. We, thus, conducted a national observational study to describe hospital admissions for KD in PLHIV in France and to identify factors associated with such admissions.

\section{PATIENTS AND METHODS}

This study was an observational retrospective multicentre study based on nationwide PMSI (Programme de Médicalisation des Systèmes d'Information) data from 2008 to 2013. The French PMSI database is similar to the Medicare database and includes all admissions in all hospitals from 1997 onwards. Since 2007, it has been possible to link all discharge abstracts for a single patient. Diagnoses identified during hospital stays are coded according to the t10th edition of the International Classification of Diseases, 10th revision (ICD-10). This database provides a vast amount of epidemiological information regarding hospitalised patients in France, ${ }^{15-18}$ is truly representative and can be used to detect rare events.

In the first part of the study, all patients older than 18 years hospitalised with a main or associated diagnosis of HIV infection (ICD-10 code B20-24, Z21) were eligible. All patients with at least one hospitalisation lasting more than 24 hours were included. Those hospitalised for less than 24 hours were excluded since repeated short hospitalisations were most often regular follow-up consultations or ambulatory care. Age, sex, comorbidities and patient outcomes in the year following hospitalisation were collected. Each patient was classified as having past or present AIDS-defining illness (Centers for Disease Control and Prevention (CDC) stage C, codes B20-22, B241) or being HIV infected without progression to AIDS (CDC stages A and B, codes B23, B240, B249 and Z21). Hospital admissions with a main diagnosis code corresponding to KD (ICD-10 codes N00-N39) in the year following the first hospitalisation with a diagnosis of HIV infection were collected. The KDs were defined and classified as follows: ARF (codes N17 and 19), CKD either at first diagnosis or with associated complications, ARF (code N18), nephrolithiasis (codes N13, 20, 21, 22 and 23), pyelonephritis (codes N10, 11, 12 and 15), renal parenchymal diseases (including glomerular and tubular diseases) (codes N01-06, N08, N14, N16 and N25), and other KD (codes N26, 27, 28, 29 and N39). For glomerular diseases, both the syndrome (eg, nephrotic syndrome) and the related disease (eg, membranous nephropathy) were available. The identified syndromes were haematuria, proteinuria, nephrotic syndrome and acute or chronic nephritic syndrome. Comorbidities were also collected: hypertension (codes I10-I15), diabetes mellitus (codes E10-E14), dyslipidaemia (code E78), heart failure (code I50), coinfection with hepatitis B and/orhepatitis C (codes B18.0 and B18.2), obesity (code E66) and cardiovascular diseases including coronary artery disease (codes I20-I25), peripheral artery disease (code I702) and stroke (codes I63, I64). The annual prevalence of hospitalisation due to KD among PLHIV and the annual distribution of the various KD were then assessed. The changes in annual prevalence were determined for the study period (2008-2013).

In the second part of the study which aimed to assess the incidence of hospital admission due to KD in PLHIV, only PLHIV newly followed in 2009 were taken into account, whatever the duration of their first hospitalisation. Newly followed PLHIV were defined as patients hospitalised with a diagnosis of HIV infection in 2009 but with no hospitalisation for HIV infection in the previous 2 years. We considered these patients as 'newly followed PLHIV' because very few patients with HIV in France had medical follow-up outside the hospital setting during the study period. The newly followed PLHIV were monitored for 5 years, and all hospitalisations for $\mathrm{KD}$ were analysed along with any associated comorbidities.

For all analyses, patients who were hospitalised several times for KD were only considered once. Changes over time in the proportion of PLHIV with KD were assessed using the Cochran-Armitage Test, and changes in the number of patients in the HIV and KD cohorts were analysed with a Poisson model. We compared the patient age in the AIDS-defining illness, non-AIDS HIV and the whole HIV cohorts with the t-test, Mann Whitney test or Kruskal-Wallis test. The characteristics of patients with and without AIDS were compared using Fisher's exact test and the $\mathrm{X}^{2}$ test. A Cox model was used to determine factors associated with hospitalisation for incident $\mathrm{KD}$, with a follow-up of 5 years. Individuals were censored at death, at the end of the follow-up or the latest all-cause hospitalisation for people without $\mathrm{KD}$. In multivariate analyses, we introduced all the variables considered significant in the univariate analyses $(p<0.20)$ and according to their clinical relevance. We have, therefore, included: age, gender, having past or present AIDS-defining illness, obesity, coinfection, dyslipidaemia, hypertension, diabetes and cardiovascular diseases in the multivariate analyses. The proportional hazards assumption was assessed for each variable and interaction tested. To limit sparse-data bias, ${ }^{19}$ we performed two penalisation estimations: the first one using the Firth bias adjustment ${ }^{20}$ and the second one using data augmentation. ${ }^{21}$ SAS statistical software (V.9.3) was used for all analyses. 
RESULTS

\section{Patient characteristics}

From 2008 to 2013, more than 5 million people were hospitalised each year in France. Their main characteristics over the study period are shown in table 1 . The proportion of patients hospitalised for $\mathrm{KD}$ rose progressively from $1.9 \%$ in 2008 to $2.4 \%$ in 2013 ( $p<0.0001$ ).

During the same period, an average of 10862 PLHIV was hospitalised per year (table 2). There was a significant decrease in the number of PLHIV admissions from 2010 to $2013(p<0.0001)$. There was also a significant increase in the mean age of these patients, from 45 years in 2008 to 48 years in 2013 .

Since the mean proportion of patients hospitalised for $\mathrm{KD}$ was globally $2.2 \%$ in the general population (table 1 ) and $3.5 \%$ in PLHIV (table 2), the prevalence of admission for KD in PLHIV was 1.5 higher than in the general population.

The comorbidities known to be associated with an increased risk of $\mathrm{KD}$ in 2009 are presented in table 3. Hepatic comorbidities were more frequently observed in hospitalised PLHIV ( $12.2 \%$ vs $0.3 \%$ ), whereas all other comorbidities were more frequent in the general hospitalised population.

\section{The distribution of hospitalisations for KD in PLHIV}

Of the HIV-infected patients admitted to hospital, 2254 $(3.5 \%)$ were hospitalised for at least 24 hours for $\mathrm{KD}$ during the 5-year study period (table 2). A significant increase was observed over time, from $3.0 \%$ in 2008 to $3.7 \%$ in 2013 ( $\mathrm{p}=0.0019)$. The patients with HIV hospitalised for $\mathrm{KD}$ were significantly older than the overall HIV cohort $(50 \pm 13$ years vs $46 \pm 12$ years, $\mathrm{p}<0.01)$.

The most common KDs on admission were ARF $(25.4 \%, \quad(23.0 \%-30.1 \%))$, nephrolithiasis $(22.1 \%$, $(20.2 \%-24.0 \%))$ and pyelonephritis $(22.1 \%$, (15.9\%$26.8 \%))$. The yearly proportion of patients hospitalised for ARF varied from $0.7 \%(n=77)$ to $1.0 \%(n=101)(0.9 \%$ on average, table 2). Among hospitalisations for $\mathrm{KD}$, the proportion of those linked to ARF remained stable over the study period $(25.4 \%(23.0 \%-30.1 \%))$. The mean age of patients hospitalised with ARF was higher than the mean age of the whole cohort $(51 \pm 13$ years vs $46 \pm 12$ years). Hospitalisation for ARF was more often observed in patients with AIDS than in PLHIV without AIDS $(30.1 \%$ vs $21.2 \%)$, the latter being older than the former $(55 \pm 12$ years vs $49 \pm 12$ ). Hospitalisation for pyelonephritis was more common in patients with AIDS, while the opposite was observed for nephrolithiasis.

Other causes of hospitalisation for $\mathrm{KD}$ were renal parenchymal diseases $(8.6 \%,(5.9 \%-11.4 \%))$, out of these glomerular diseases $(6.4 \%$ of the hospitalisations for $\mathrm{KD})$, with a roughly stable proportion throughout the study period. Of these glomerular diseases, 27\% $(n=48)$ were defined only by their glomerular syndrome, $25.2 \% \quad(n=45)$ were defined via their association with another disease (such as infectious diseases, systemic disease and diabetes mellitus), and only $47.8 \% \quad(\mathrm{n}=85)$ were histologically

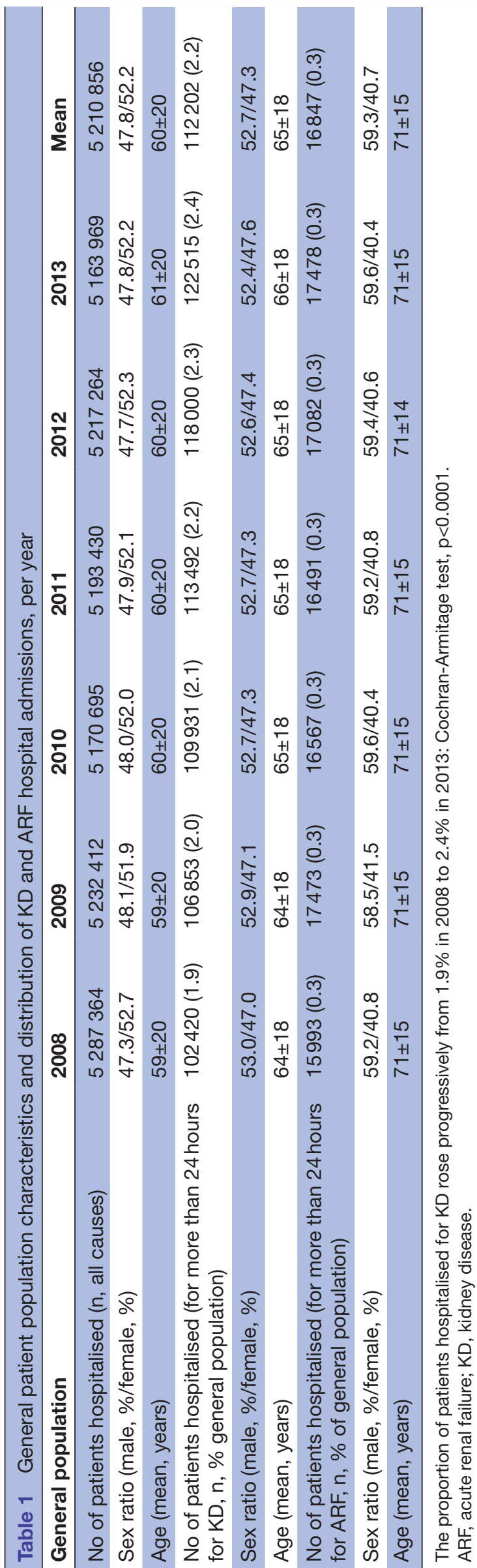




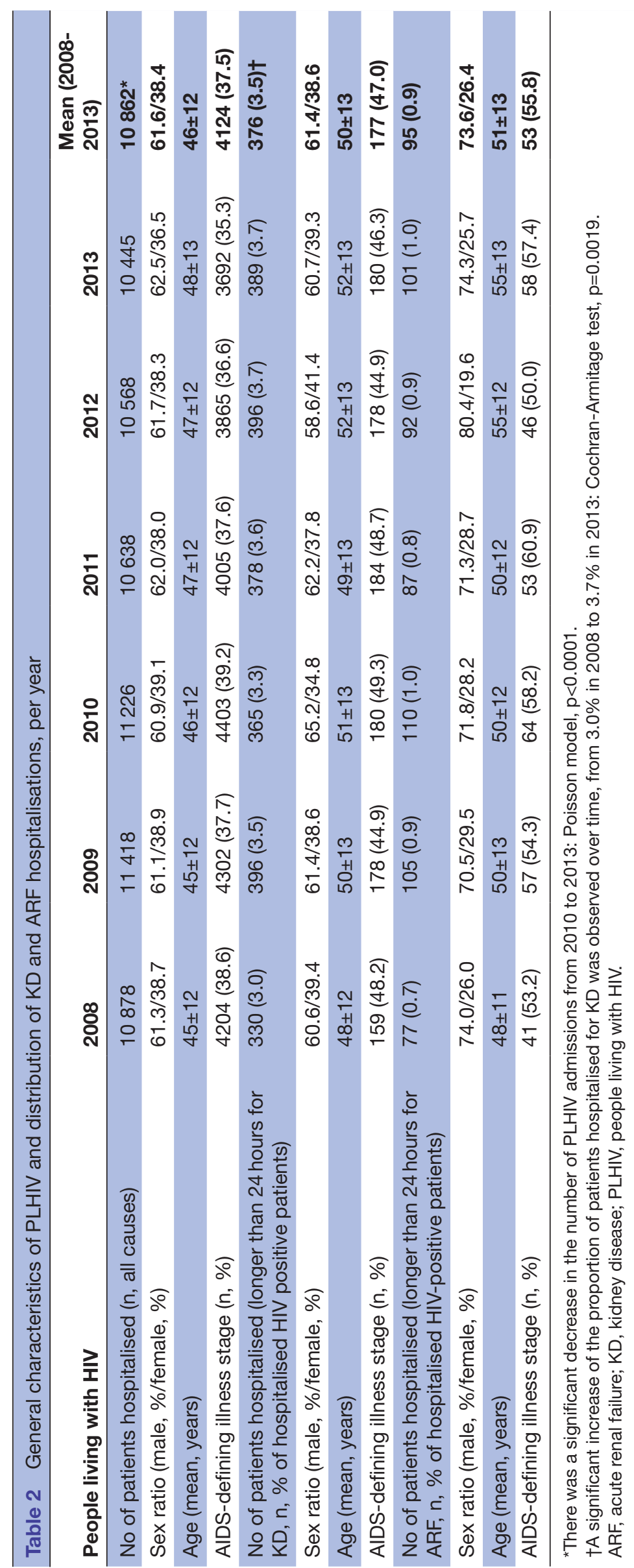


Table 3 Comorbidities in the hospitalised general population and hospitalised HIV population for the year 2009

\begin{tabular}{|c|c|c|c|}
\hline Comorbidities & $\begin{array}{l}\text { General population } \\
n=5232412\end{array}$ & HIV $n=11418$ & P value* \\
\hline Hepatitis coinfections $\uparrow, n, \%$ & $16390(0.3)$ & $1397(12.2)$ & $<0.0001$ \\
\hline Diabetes mellitus, $n, \%$ & $526511(10.1)$ & $651(5.7)$ & $<0.0001$ \\
\hline Cardiovascular diseasesł, n, \% & $683316(13.1)$ & $821(7.2)$ & $<0.0001$ \\
\hline Heart failure, $n, \%$ & 218697 (4.2) & $151(1.3)$ & $<0.0001$ \\
\hline
\end{tabular}

${ }^{*} X^{2}$ test.

†Hepatitis coinfections included hepatitis $C$ and/or hepatitis B.

‡Cardiovascular diseases included coronary artery diseases, peripheral artery disease and stroke.

assessed. The proportion of renal biopsies in AIDS PLHIV was similar to that in non-AIDS PLHIV ( $45.5 \%$ vs $37.6 \%$ ). Of the histologically assessed glomerular diseases, FSGS was diagnosed in $37.6 \%$ of cases. While there was a non-significant upwards trend in the use of renal biopsy over the study period, the proportion of FSGS declined from $63.6 \%$ in 2008 to $27.3 \%$ in 2013 . Other glomerular diseases were membranous nephropathy $(14.1 \%, \mathrm{n}=12)$, crescentic glomerulonephritis $(12.9 \%, \mathrm{n}=11)$, undetermined glomerulonephritis $(10.6 \%, \mathrm{n}=9)$, minimal change disease $(9.4 \%, \mathrm{n}=8)$ and membranoproliferative glomerulonephritis $(7.1 \%, \mathrm{n}=6)$. Mesangial proliferative glomerulonephritis and postinfectious glomerulonephritis were found in less than $5 \%$. Tubulointerstitial disorders were infrequent $(2.2 \%, \mathrm{n}=49)$, decreasing from $3.3 \%$ in 2008 to $1 \%$ in 2013 ( $\mathrm{p}=0.18)$. Drug-related tubulointerstitial or tubular injuries were the main cause of these hospitalisations $(53.1 \%, \mathrm{n}=26)$.

\section{Hospitalisations for KD in PLHIV newly followed in 2009}

Comorbidities in all newly followed PLHIV and in those hospitalised for $\mathrm{KD}$ are summarised in table 4 .

In 2009, 1113 PLHIV were newly followed, 66 of which were hospitalised for KD during the entire follow-up, which reveals a 5-year cumulative incidence of 5.9\% (figure 1). Twenty-two $(33.0 \%)$ of these patients were coded for $\mathrm{KD}$ at the initial hospitalisation or within 1 year.

The median of follow-up time was 843 days with an IQR of 1459 days. As there was an interaction between having past or present AIDS-defining illness, or not, and gender, Kaplan-Meier curves and the associated log-rank tests for each gender are presented in figure 2A,B. For women, the risk of KD was higher for those having past or present AIDS-defining illness than those who did not ( $\mathrm{p}=0.0127)$. In multivariate survival analysis (table 5), cardiovascular disease was significantly associated with the risk of KD (HR 3.39, 95\% CI 1.22 to 9.42). When focusing on female gender, having past or present AIDS-defining illness remained associated with this risk of $\mathrm{KD}$ (HR 3.67, 95\% CI 1.26 to 10.70). As our CIs were wide with huge upper $95 \%$ limits (greater about 10), we performed two penalisation estimations: the first one using the Firth bias adjustment and the second one using data augmentation. With the Firth bias adjustment, we observed a slight decrease of the upper $95 \%$ limits, while with the penalisation by data augmentation, these limits were widely reduced with an HR 3.30 (95\% CI 1.46 to 7.49) for cardiovascular disease

Table 4 Comorbidities in all newly followed PLHIV and in those hospitalised for KD

\begin{tabular}{|c|c|c|c|}
\hline Comorbidities & $\begin{array}{l}\text { Total newly followed PLHIV } \\
n=1113\end{array}$ & $\begin{array}{l}\text { Newly followed PLHIV } \\
\text { with KD } n=66\end{array}$ & $p$ value* \\
\hline Hepatitis co-infections, $n, \%$ & $89(8.0)$ & $4(6.1)$ & 0.5708 \\
\hline Diabetes mellitus , n, \% & $37(3.3)$ & $2(3.0)$ & 1.0000 \\
\hline Cardiovascular diseases $†, n, \%$ & $28(2.5)$ & $5(7.6)$ & 0.0330 \\
\hline Heart failure, n, \% & $5(0.4)$ & $1(1.5)$ & 0.2928 \\
\hline
\end{tabular}

${ }^{*}$ Comparison between newly followed PLHIV hospitalised with KD and those without, Fisher's exact test or $\chi^{2}$.

†Cardiovascular diseases included coronary artery diseases, peripheral artery disease and stroke.

†Hepatitis coinfections including hepatitis $\mathrm{C}$ and/or hepatitis $\mathrm{B}$.

$\mathrm{KD}$, kidney disease; PLHIV, people living with HIV. 


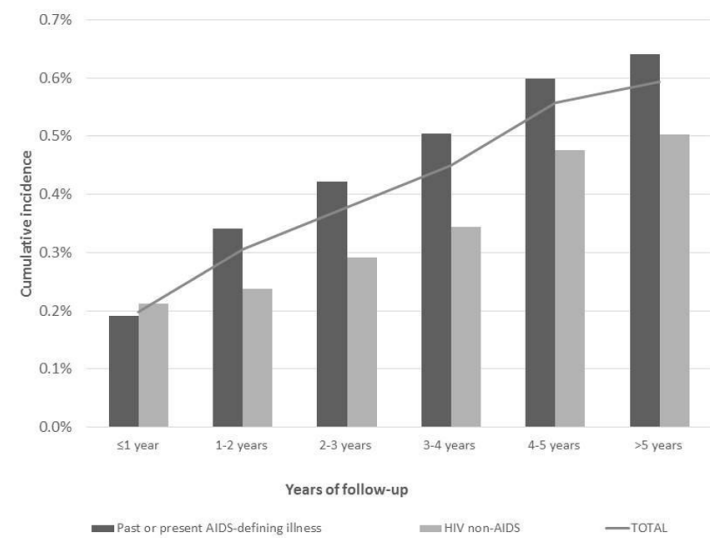

Figure 1 Cumulative incidence of $K D$ requiring hospitalisation for all newly followed PLHIV (grey curve), for those without past or present AIDS (light bars) and for those with past or present AIDS-defining illness (dark bars). KD, kidney disease; PLHIV, people living with HIV.

and an HR 2.45 (95\% CI 1.07 to 5.58) for female gender having past or present AIDS-defining illness (table 5).

Among the causes of incident KD requiring hospital admission, ARF was involved in 25 cases, and $66.7 \%$ of
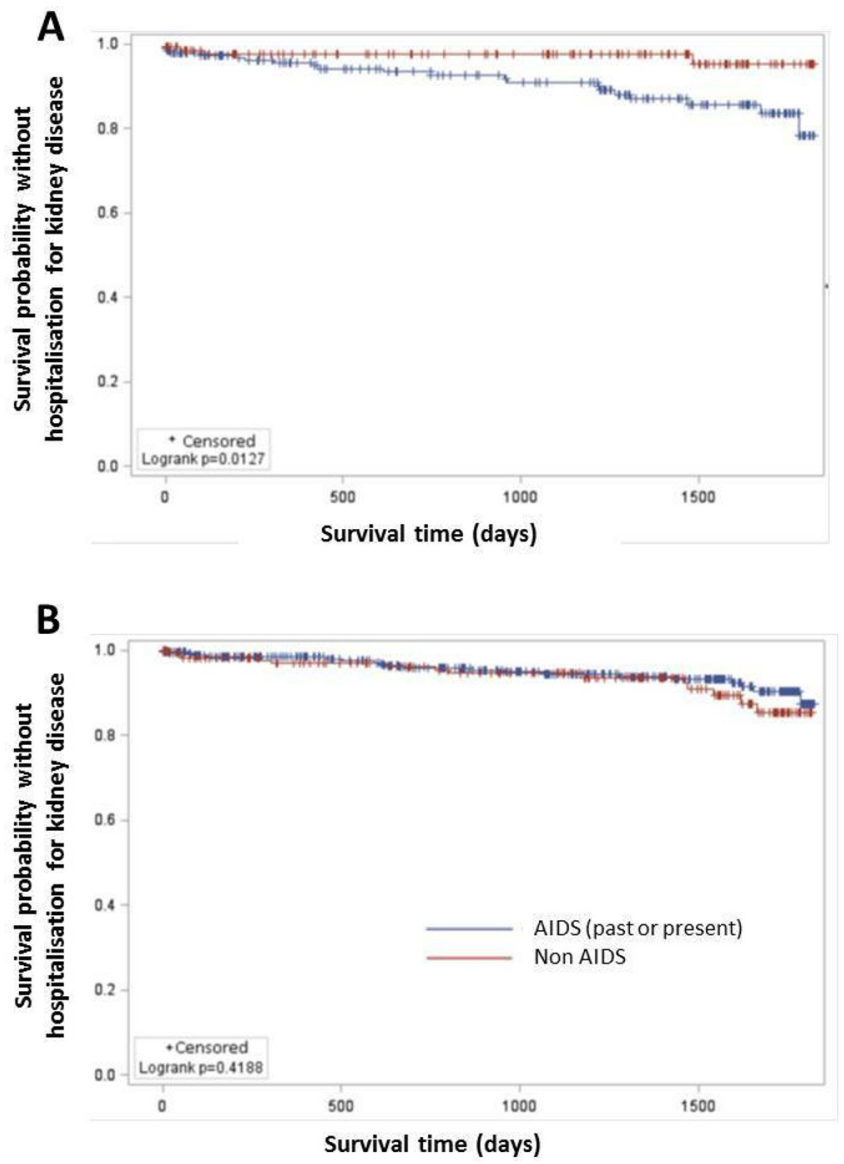

Figure 2 Kaplan-Meier curve to estimate the risk of kidney disease with time according to the AIDS status (having past or present AIDS-defining illness or not) (A) for women, (B) for men. these occurred in the first 2 years of follow-up $(50.0 \%$ in the first year and $16.7 \%$ in the second year). The 5 -year cumulative incidence of ARF was $1.6 \%$. No risk factor was significantly associated with the development of incident ARF requiring hospitalisation.

\section{DISCUSSION}

First, this study found that patients hospitalised for $\mathrm{KD}$ are 1.5 more frequent in PLHIV than in the general population. The rate of patients hospitalised increased steadily over time despite the fact that risk factors for $\mathrm{KD}$ are less frequent in PLHIV than in the general hospitalised population, except for hepatitis coinfections. These rates have increased significantly in recent years (from 3.0\% in 2008 to $3.7 \%$ in 2013), although similar to the rates observed in the general population (5-year difference $0.7 \%$ vs $0.5 \%$ ). ARF, nephrolithiasis and pyelonephritis accounted for more than three-quarters of hospital admissions for KD in our study. Glomerular or tubular diseases were rather infrequent but stable causes of hospitalisation. The most frequent glomerular disease was FSGS with $37.6 \%$ of histological codes, although with a downward trend since the beginning of the HAART era.

Another point is that ARF often occurs within 2 years following the diagnosis of HIV for the two-thirds of the newly diagnosed and followed PLHIV who subsequently presented ARF.

The last key finding is that nearly $6 \%$ of PLHIV were hospitalised for KD during the 5 years following the diagnosis of HIV infection, and that the risk was strongly associated with the presence of cardiovascular disease.

To our knowledge, this study is the first to focus on hospital admissions for $\mathrm{KD}$ in a large population of PLHIV. In our study, more than 10850 PLHIV were hospitalised each year, that is, about $10 \%$ of the estimated cohort of 110900 PLHIV medically followed up in 2010 in France. The exhaustive nature of our data ensures that the results are representative of all hospitalisations for $\mathrm{KD}$ in France, which is particularly important for both HIV and $\mathrm{KD}$ which can be influenced by the ethnic origins of the population

This study is the first to show such a trend in a nationwide setting, and is consistent with other studies that have reported hospitalisations rates for $\mathrm{KD}$ ranging from $2.6 \%$ to $3.3 \%{ }^{22}$

The proportion of FSGS at the beginning of our study $(63.6 \%$ in 2008$)$ is similar to that reported in other studies, ${ }^{132324}$ and the trend towards a decrease in the percentage we observed $(27.3 \%$ in 2013$)$ was also reported in another recent study $(28 \%))^{25}$

ARF was responsible for approximately $1 \%$ of in hospitalised PLHIV, a rate which is lower than that observed in the few previous studies focusing on PLHIV (2.8-5.9 per 100 patient-years. ${ }^{3426}$ The most likely explanation is that we only considered ARF cases leading to hospitalisation, and not all ARF, including those occurring in inpatients hospitalised for other reasons (eg, sepsis or volume 


\begin{tabular}{|c|c|c|c|}
\hline & Cox model & Firth bias adjustment & Penalisation by data augmentation \\
\hline & HR (95\% Cl) & HR $(95 \% \mathrm{Cl})$ & HR (95\% Cl) \\
\hline Cardiovascular disease & 3.39 (1.22 to 9.42$)$ & 3.77 (1.23 to 8.91$)$ & 3.30 (1.46 to 7.49$)$ \\
\hline AIDS $^{*}$ in men & $0.72(0.37$ to 1.40$)$ & 0.71 (0.36 to 1.39$)$ & $0.93(0.51$ to 1.72$)$ \\
\hline
\end{tabular}

Adjusted on age, obesity, coinfection, dyslipidaemia, hypertension and diabetes.

${ }^{*}$ AIDS, having past or present AIDS-defining illness.

depletion). For example, $31 \%$ (34/111) of patients in the study by Franceschini et al developed ARF during a hospital stay, and $38 \%$ had community-acquired ARF not requiring hospitalisation. ${ }^{27}$ Furthermore, we also did not consider ARF in PLHIV hospitalised for CKD. This also probably explains why many studies reported ARF hospitalisation rates that were 3 or 4 times higher in PLHIV than in the general population, whereas we observed an ARF hospitalisation rate in PLHIV close to that observed in the general population $(0.6 \%-1 \%) \cdot{ }^{28}{ }^{29}$ Nonetheless, ARF remains a concern in PLHIV as the average age at the time of hospitalisation is lower than that in the general population.

Our study has several limitations, the main one being the use of administrative codes. However, these codes are based on the clinical judgement of physicians and laboratory values. The use of this type of database has been validated for $\mathrm{KD}^{30}{ }^{31}$ and particularly for $\mathrm{ARF}^{30}{ }^{31} \mathrm{In}$ addition, the infrequent use of renal biopsies precluded an extended analysis of parenchymental KD leading to hospitalisation, and we do not distinguish HIVAN from HIVICK. Finally, it was not possible to provide accurate characteristics of the HIV infection in the absence of data on HIV viral load or CD4 cell count, and/or on the antiretroviral drugs used. Nonetheless, the aim of our study was to provide an overview of KD in PLHIV. Moreover, our high HRs and wide CIs could be a sign of sparse-data bias, which may be due to our small number of events. However, after performing penalised estimation such as Firth bias adjustment or penalisation by data augmentation, we were able to reduce our upper $95 \%$ limits while maintaining the significance of our different factors.

Other studies are necessary to extend the available data about each kidney condition in PLHIV.

FSGS, which is probably represented by HIVAN, is still the most frequent nephropathy in PLHIV though it has decreased relative to other glomerulopathies following the expansion of HAART. ${ }^{13}{ }^{15}$ Nevertheless, nearly $60 \%$ of our PLHIV cohort hospitalised for glomerular disease were not biopsied. Almost $50 \%$ of these patients had proteinuria or nephrotic syndrome $(3.4 \%$ and $44.4 \%$, respectively). These figures reveal a gap between the proportion of glomerular diseases and the number of renal biopsies performed. Several studies have confirmed the importance of performing kidney biopsies in PLHIV. $^{25}{ }^{32}$ Radiological examinations, urinary findings, renal function biological and proteinuria evaluations do not provide sufficiently specific information to diagnose HIV-related nephropathy. ${ }^{32}$ Much like US guidelines from $2014,{ }^{4}$ the French guidelines ${ }^{34}$ recommend referring patients toe a nephrologist if the estimated glomerular filtration rate falls to less than $60 \mathrm{~mL} / \mathrm{min}$ and/or as soon as proteinuria appears. The US recommendations also suggest that biopsies should be done in HIV-infected patients in whom a definitive diagnosis may affect management or inform the prognosis. ${ }^{4}$

ARF often occurs early during follow-up ${ }^{35} 36$ with a 10-fold higher rate of ARF in the first 3 months after the introduction of HAART, ${ }^{35}$ probably reflecting the disease burden in PLHIV with more advanced HIV disease and concurrent infections at the time of admission. 3536 Indeed, opportunistic infections may play an important role in the development of early-onset ARF.

By contrast, associated comorbidities and HAART toxicity are important factors leading to 'late-onset $\mathrm{ARF},{ }^{35}$ as suggested by the older age of non-AIDS PLHIV experiencing ARF. This can probably be explained by the pathophysiological link between some comorbidities such a cardiovascular disease and $\mathrm{KD}$, that has also been reported in PLHIV. ${ }^{37-39}$ Since cardiovascular comorbidity is particularly frequent in PLHIV, ${ }^{40}$ it should be closely monitored and managed to prevent serious KD.

In conclusion, the proportion of PLHIV hospitalised for ARF and other types of KD remains significant and has not decreased despite the increasing use of HAART. PLHIV must be considered a high-risk population for $\mathrm{KD}$, particularly for those with simultaneous cardiovascular disease. ARF remains the leading cause of hospitalisation for renal disorders, which essentially occur early during the follow-up, suggesting the need for regular and specific follow-up, at least during the first year. Glomerular and tubular diseases, which were also stable over time, should be documented more often by biopsies, whose results can inform the clinicians who treat these diseases and improve prognostic outcomes. All of these data reflect changes in the impact of HIV infection, comorbidities and treatments, and underline the need to regularly address this issue in the future.

Contributors ML and LP conceptualised and designed the study, interpreted the data and wrote the paper. JC performed the data analysis. AS-R and CM contributed substantially to writing the manuscript. MB, P-HB J-MR and PC participated in the interpretation of the results and reviewed and revised the manuscript drafts. $C Q$ oversaw the data analysis and interpretation and contributed substantially to writing the manuscript. All authors accept responsibility for the paper as published. 
Funding The authors have not declared a specific grant for this research from any funding agency in the public, commercial or not-for-profit sectors.

Competing interests None declared.

Patient consent for publication Not required.

Ethics approval This study was approved by the National Committee for data protection (registration number 1576793) and therefore was conducted in accordance with the Declaration of Helsinki. The PMSI database was transmitted by the national agency for the management of hospitalisation data (ATIH number 2015-111111-47-33).

Provenance and peer review Not commissioned; externally peer reviewed.

Data sharing statement The PMSI database was transmitted by the national agency for the management of hospitalisation data. The use of these data by our department was approved by the National Committee for data protection. We are not allowed to transmit these data.

Open access This is an open access article distributed in accordance with the Creative Commons Attribution Non Commercial (CC BY-NC 4.0) license, which permits others to distribute, remix, adapt, build upon this work non-commercially, and license their derivative works on different terms, provided the original work is properly cited, appropriate credit is given, any changes made indicated, and the use is non-commercial. See: http://creativecommons.org/licenses/by-nc/4.0/.

\section{REFERENCES}

1. Selik RM, Byers RH, Dworkin MS. Trends in diseases reported on U.S. death certificates that mentioned HIV infection, 1987-1999. J Acquir Immune Defic Syndr 2002;29:378-87.

2. Adih WK, Selik RM, Hu X. Trends in diseases reported on US death certificates that mentioned HIV infection, 1996-2006. J Int Assoc Physicians AIDS Care 2011;10:5-11.

3. Wyatt CM, Arons RR, Klotman PE, et al. Acute renal failure in hospitalized patients with HIV: risk factors and impact on in-hospital mortality. AIDS 2006;20:561-5.

4. Lucas GM, Ross MJ, Stock PG, et al. Clinical practice guideline for the management of chronic kidney disease in patients infected with HIV: 2014 update by the HIV Medicine Association of the Infectious Diseases Society of America. Clin Infect Dis 2014;59:e96-e138.

5. Li Y, Shlipak MG, Grunfeld C, et al. Incidence and risk factors for acute kidney injury in HIV Infection. Am J Nephrol 2012;35:327-34.

6. Choi Al, Li Y, Parikh C, et al. Long-term clinical consequences of acute kidney injury in the HIV-infected. Kidney Int 2010;78:478-85.

7. Hsu CY, Chertow GM, McCulloch CE, et al. Nonrecovery of kidney function and death after acute on chronic renal failure. Clin J Am Soc Nephrol 2009;4:891-8.

8. Lattanzio MR, Kopyt NP. Acute kidney injury: new concepts in definition, diagnosis, pathophysiology, and treatment. J Am Osteopath Assoc 2009;109:13-19.

9. Lucas GM, Mehta SH, Atta MG, et al. End-stage renal disease and chronic kidney disease in a cohort of African-American HIV-infected and at-risk HIV-seronegative participants followed between 1988 and 2004. AIDS 2007;21:2435-43.

10. Mocroft A, Lundgren JD, Ross M, et al. Cumulative and current exposure to potentially nephrotoxic antiretrovirals and development of chronic kidney disease in HIV-positive individuals with a normal baseline estimated glomerular filtration rate: a prospective international cohort study. Lancet HIV 2016;3:e23-e32.

11. Déti EK, Thiébaut $R$, Bonnet $F$, et al. Prevalence and factors associated with renal impairment in HIV-infected patients, ANRS C03 Aquitaine Cohort, France. HIV Med 2010;11:308-17.

12. Wali RK, Drachenberg CI, Papadimitriou JC, et al. HIV-1-associated nephropathy and response to highly-active antiretroviral therapy. Lancet 1998;352:783-4.

13. Lescure FX, Flateau C, Pacanowski J, et al. HIV-associated kidney glomerular diseases: changes with time and HAART. Nephrol Dial Transplant 2012;27:2349-55.

14. Flandre P, Pugliese $P$, Cuzin L, et al. Risk factors of chronic kidney disease in HIV-infected patients. Clin J Am Soc Nephrol 2011;6:1700-7.

15. Pagès PB, Cottenet J, Mariet AS, et al. In-hospital mortality following lung cancer resection: nationwide administrative database. Eur Respir J 2016;47:1809-17.
16. Creuzot-Garcher C, Benzenine E, Mariet AS, et al. Incidence of acute postoperative endophthalmitis after cataract surgery: a nationwide study in France from 2005 to 2014. Ophthalmology 2016;123:1414-20.

17. Abdulmalak C, Cottenet J, Beltramo G, et al. Haemoptysis in adults: a 5-year study using the French nationwide hospital administrative database. Eur Respir J 2015;46:503-11.

18. Quantin C, Benzenine E, Velten M, et al. Self-controlled case series and misclassification bias induced by case selection from administrative hospital databases: application to febrile convulsions in pediatric vaccine pharmacoepidemiology. Am J Epidemiol 2013;178:1731-9.

19. Greenland S, Mansournia MA, Altman DG. Sparse data bias: a problem hiding in plain sight. BMJ 2016;352:i1981.

20. Firth D. Bias reduction of maximum likelihood estimates. Biometrika 1993;80:27-38.

21. Sullivan SG, Greenland S. Bayesian regression in SAS software. Int $J$ Epidemiol 2013;42:308-17.

22. Crum-Cianflone NF, Grandits G, Echols S, et al. Trends and causes of hospitalizations among HIV-infected persons during the late HAART era: what is the impact of CD4 counts and HAART use? J Acquir Immune Defic Syndr 2010;54:248-57.

23. Berliner AR, Fine DM, Lucas GM, et al. Observations on a cohort of HIV-infected patients undergoing native renal biopsy. Am J Nephrol 2008;28:478-86.

24. Bohmart A, Burns G. Renal disease in an urban HIV population in the era prior and following the introduction of highly active antiretroviral therapy. J Natl Med Assoc 2011;103:513-7.

25. da Silva DR, Gluz IC, Kurz J, et al. Multiple facets of HIV-associated renal disease. Braz J Med Biol Res 2016;49:e5176.

26. Ibrahim F, Naftalin C, Cheserem E, et al. Immunodeficiency and renal impairment are risk factors for HIV-associated acute renal failure. AIDS 2010;24:2239-44.

27. Franceschini N, Napravnik S, Eron JJ, et al. Incidence and etiology of acute renal failure among ambulatory HIV-infected patients. Kidney Int 2005;67:1526-31.

28. Schissler MM, Zaidi S, Kumar H, et al. Characteristics and outcomes in community-acquired versus hospital-acquired acute kidney injury. Nephrology 2013;18:183-7.

29. Mesropian PD, Othersen J, Mason D, et al. Community-acquired acute kidney injury: A challenge and opportunity for primary care in kidney health. Nephrology 2016;21:729-35.

30. Vlasschaert ME, Bejaimal SA, Hackam DG, et al. Validity of administrative database coding for kidney disease: a systematic review. Am J Kidney Dis 2011;57:29-43.

31. Waikar SS, Wald R, Chertow GM, et al. Validity of international classification of diseases, ninth revision, clinical modification codes for acute renal failure. J Am Soc Nephrol 2006;17:1688-94.

32. Cohen SD, Kimmel PL. Renal biopsy is necessary for the diagnosis of HIV-associated renal diseases. Nat Clin Pract Nephrol 2009;5:22-3.

33. Fine DM, Perazella MA, Lucas GM, et al. Kidney biopsy in HIV: beyond HIV-associated nephropathy. Am J Kidney Dis 2008;51:504-14.

34. Morlat P. Prise en charge médicale des personnes vivant avec le VIH: recommandations du groupe d'experts: rapport 2013. Paris: Direction de l'information légale et administrative, 2013.

35. Roe J, Campbell LJ, Ibrahim F, et al. HIV care and the incidence of acute renal failure. Clin Infect Dis 2008;47:242-9.

36. Naicker S, Aboud O, Gharbi MB. Epidemiology of acute kidney injury in Africa. Semin Nephrol 2008;28:348-53.

37. Roy SK, Estrella MM, Darilay AT, et al. Glomerular filtration rate and proteinuria associations with coronary artery calcium among HIVinfected and HIV-uninfected men in the Multicenter AIDS Cohort Study. Coron Artery Dis 2017;28:17-22.

38. Ryom L, Mocroft A, Kirk O, et al. Predictors of advanced chronic kidney disease and end-stage renal disease in HIV-positive persons. AIDS 2014;28:187-99.

39. Ryom L, Lundgren JD, Ross M, et al. Renal Impairment and Cardiovascular Disease in HIV-Positive Individuals: The D:A:D Study. $J$ Infect Dis 2016;214:1212-20.

40. Hanna DB, Ramaswamy C, Kaplan RC, et al. Trends in Cardiovascular Disease Mortality Among Persons With HIV in New York City, 2001-2012. Clin Infect Dis 2016;63:1122-9. 